\title{
NUCLEOSYNTHESIS IN INTERMEDIATE-MASS STARS
}

\author{
JOHN C. LATTANZIO AND CHERYL A. FROST \\ Department of Mathematics, Monash University \\ Clayton, Australia \\ ROBERT C. CANNON \\ School of Biological Sciences, Southampton University \\ Southampton, England \\ AND \\ PETER R. WOOD \\ Mount Stromlo and Siding Springs Observatories \\ Australian National University, Canberra, Australia
}

\begin{abstract}
We discuss nucleosynthesis within $6 M_{\odot}$ models with $Z=0.02$, 0.008 and 0.004 . The emphasis is on the AGB phase of evolution, with particular reference to thermal pulses and Hot Bottom Burning. We find strong $\mathrm{CN}$ cycling, with substantial $\mathrm{Al}$ production, especially at low metallicities.
\end{abstract}

\section{Introduction and Method}

Our knowledge of AGB stars has increased dramatically recently, driven primarily by observational studies, with theory lagging somewhat behind. Studies are desperately needed of the nucleosynthesis which occurs during thermally pulsing evolution and particularly "Hot Bottom Burning" (hereafter HBB), where the bottom of the convective envelope of a star reaches temperatures sufficiently high for significant nucleosynthesis to take place. Thus there is a thin layer at high temperatures, and envelope material is mixed through this region and then into the outer envelope. For nuclear reactions with time-scales no longer than the mixing time-scale, we cannot make the usual assumption of instantaneous mixing, and an average burning rate. The historical motivation for realising the importance of $\mathrm{HBB}$ has been briefly summarised in Lattanzio et al. (1996) and is not repeated here. We emphasise the importance of recent ion-probe studies of mete- 
orites which reveal unusual isotopic compositions in some grains (such as $\mathrm{SiC}$ ) which were almost certainly formed in the envelopes of AGB stars.

Our study requires both detailed nucleosynthesis and evolution models, with a time-dependent mixing algorithm. We have chosen to do this with two separate calculations. First we perform the evolutionary calculations with the Mt. Stromlo evolution code, modified to include the OPAL opacities (Rogers \& Iglesias 1992). Following this, a nucleosynthesis code is used to calculate the detailed nuclear burning of 74 species with the stellar structure as previously calculated by the evolution code. Time-dependent mixing is included at this stage, in the way described by Cannon (1993). Nuclear reaction rates are primarily taken from the data base of Thielemann et al. (1987). There are some notable exceptions, such as the recent $\alpha-$ particle capture rate for ${ }^{18} \mathrm{O}$ (Käppeler et al. 1994). Details of both the code and the nuclear reaction rates may be found in Cannon et al. (1998).

\section{Pre-AGB Evolution}

The evolution of our models is qualitatively well understood, so we concentrate on composition changes. We will discuss the $M=6 M_{\odot}, Z=0.02$ case (hereafter referred to as M6Z02) and then draw comparisons with other $Z$. (All masses given are in units of $M_{\odot}$ ).

\subsection{CORE HYDROGEN BURNING AND FIRST DREDGE-UP}

Following core hydrogen exhaustion, all models show similar composition profiles to the M6Z02 case seen in Figure 1. As we move inward from the surface, the increasing temperature starts the $\mathrm{CN}$ cycle, and we see an increase in ${ }^{13} \mathrm{C}$ at the cost of ${ }^{12} \mathrm{C}$. The same region shows a decrease in ${ }^{15} \mathrm{~N}$ due to ${ }^{15} \mathrm{~N}(\mathrm{p}, \alpha){ }^{12} \mathrm{C}$, and then a slight increase due to ${ }^{18} \mathrm{O}(\mathrm{p}, \alpha){ }^{15} \mathrm{~N}$. Just slightly interior to $M=3$ the ${ }^{13} \mathrm{C}$ is itself destroyed in the production of ${ }^{14} \mathrm{~N}$, which now starts to rise. From here inwards the carbon isotopes are in equilibrium. Interior to $M \simeq 2.5$ we see substantial destruction of ${ }^{18} \mathrm{O}$ from the proton captures described above. At $M \simeq 1.8$ we see the production of ${ }^{17} \mathrm{O}$ from proton captures on ${ }^{16} \mathrm{O}$, as the $\mathrm{ON}$ cycle starts to function more strongly. As the star ascends the first giant branch the convective envelope reaches down to $M=1.256$ with consequent mixing from this point outward. This is known as the First Dredge-Up, and the key abundance changes are listed in Table 1.

\subsection{CORE HELIUM BURNING AND SECOND DREDGE-UP}

Within the helium core the star produces ${ }^{12} \mathrm{C}$ and ${ }^{16} \mathrm{O}$. The abundant ${ }^{14} \mathrm{~N}$ left over from CNO cycling means that ${ }^{14} \mathrm{~N}(\alpha, \gamma){ }^{18} \mathrm{~F}\left(\beta^{+} \nu\right){ }^{18} \mathrm{O}$ produces ${ }^{18} \mathrm{O}$ 


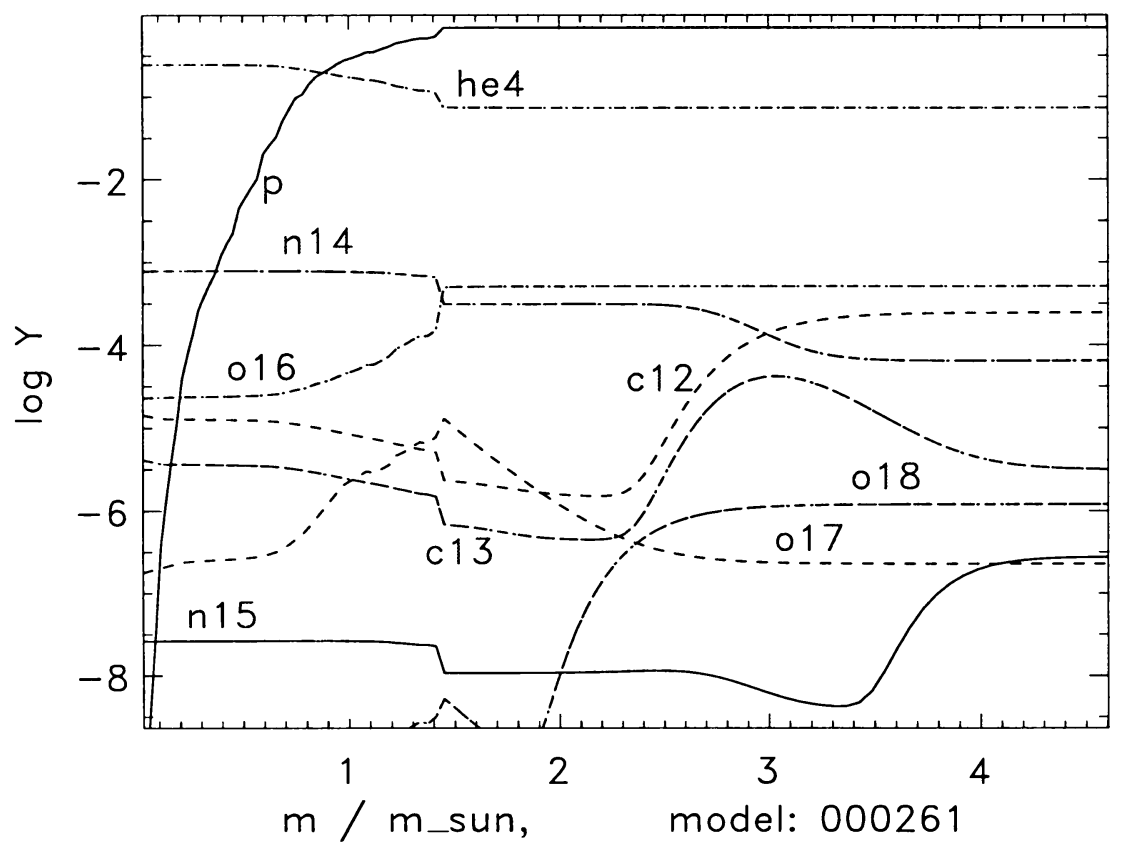

Figure 1. Composition profiles at the time of core hydrogen exhaustion for the M6Z02 model. Note that during first dredge-up the convective envelope will reach down to $M / M_{\odot}=1.256$, thus homogenising the material from that point to the surface.

TABLE 1. Selected isotopic ratios after the first (FDU) and second (SDU) dredge-up events.

\begin{tabular}{lllll}
\hline Ratio & Initial & $\begin{array}{l}Z=0.02 \\
\text { FDU,SDU }\end{array}$ & $\begin{array}{l}Z=0.008 \\
\text { FDU,SDU }\end{array}$ & $\begin{array}{l}Z=0.004 \\
\text { FDU,SDU }\end{array}$ \\
\hline${ }^{12} \mathrm{C} /{ }^{13} \mathrm{C}$ & 90 & $19.2,18.0$ & $18.8,18.1$ & $18.8,17.8$ \\
${ }^{14} \mathrm{~N} /{ }^{15} \mathrm{~N}$ & 273 & 1297,1931 & 1122,2009 & 881,2327 \\
${ }^{16} \mathrm{O} /{ }^{17} \mathrm{O}$ & 2632 & 453,408 & 708,340 & 1746,327 \\
${ }^{16} \mathrm{O} /{ }^{18} \mathrm{O}$ & 500 & 562,572 & 562,590 & 501,597 \\
\hline
\end{tabular}

and then ${ }^{18} \mathrm{O}(\alpha, \gamma){ }^{22} \mathrm{Ne}$ which produces some neutrons via ${ }^{22} \mathrm{Ne}(\alpha, \mathrm{n}){ }^{25} \mathrm{Mg}$. The hydrogen shell burning shows the usual signature of the CNO cycles, much as seen in Figure 1. The nucleosynthesis between the hydrogen "exhausted" region and the edge of the convective helium core mimics that which we will later see in the intershell region on the AGB. Outside the convective core the ${ }^{13} \mathrm{C}$ is destroyed by $(\alpha, \mathrm{n})$ and produces neutrons, which 
feed (n,p) reactions on ${ }^{14} \mathrm{~N}$ to produce ${ }^{14} \mathrm{C}$. Thus we see an increase in ${ }^{14} \mathrm{C}$ and in the protons. Further inward the ${ }^{14} \mathrm{C}$ has decayed into ${ }^{14} \mathrm{~N}$.

\section{Early AGB Evolution}

The second dredge-up phase occurs early on the AGB. Table 1 gives some abundance ratios, but note that for $6 M_{\odot}$ there is the complication that HBB begins during second dredge-up. After second dredge-up the star begins pulsing, which drives convection from the helium shell almost to the hydrogen shell (for details see Frost \& Lattanzio 1996b). Later, envelope convection extends inward and penetrates the carbon-rich region after the $7^{\text {th }}$ pulse for M6Z02, the $6^{\text {th }}$ pulse for M6Z008 and the $5^{\text {th }}$ pulse for M6Z004. This periodic third dredge-up becomes very deep, and we soon find the dredge-up parameter $\lambda \simeq 0.9$ (see also Vassiliadis \& Wood 1993). This is discussed further in Frost \& Lattanzio (1996a).

With the onset of HBB the Cameron-Fowler beryllium transport mechanism produces large amounts of ${ }^{7} \mathrm{Li}$ (see Boothroyd \& Sackmann 1992). Our results are in agreement with these authors, to whom we defer for details.

The carbon-rich convective pocket mixes the products of helium burning throughout the intershell region, and at the next dredge-up the products of this nucleosynthesis are mixed to the surface. This pocket contains a relatively large amount of ${ }^{22} \mathrm{Ne}$ at the cost of ${ }^{14} \mathrm{~N}$ and ${ }^{18} \mathrm{O}$. Also produced in the intershell zone is ${ }^{19} \mathrm{~F}$ from alpha captures on ${ }^{15} \mathrm{~N}$, which is also dredged to the surface following each thermal pulse.

\section{Advanced AGB Evolution}

As the star ascends the AGB the temperature at the base of the convective envelope increases dramatically, reaching values of up to 80 or 90 million $\mathrm{K}$. This causes $\mathrm{CNO}$ cycling as well as processing by the $\mathrm{Ne}-\mathrm{Na}$ and $\mathrm{Mg}-\mathrm{Al}$ cycles. Selected isotopic ratios are shown in Figure 2 for the thermally pulsing phase of the M6Z02 model. Mass loss (included here from Vassiliadis \& Wood 1993) decreases the mass of the envelope, and eventually this shuts off the HBB. For M6Z02 this happens long before the third dredge-up ends (calculations for other models have not proceeded this far, yet) when the envelope mass is about $2.4 M_{\odot}$. The details of the surface composition at this stage depend critically on the competition between these effects (e.g. Groenewegen \& de Jong 1993). Thus, for most of its lifetime on the AGB, HBB prevents the M6Z02 model from becoming a carbon star. But when HBB ends, the third dredge-up may yet produce a carbon star (we will address this in a later paper). We now look in more detail at the nucleo- 


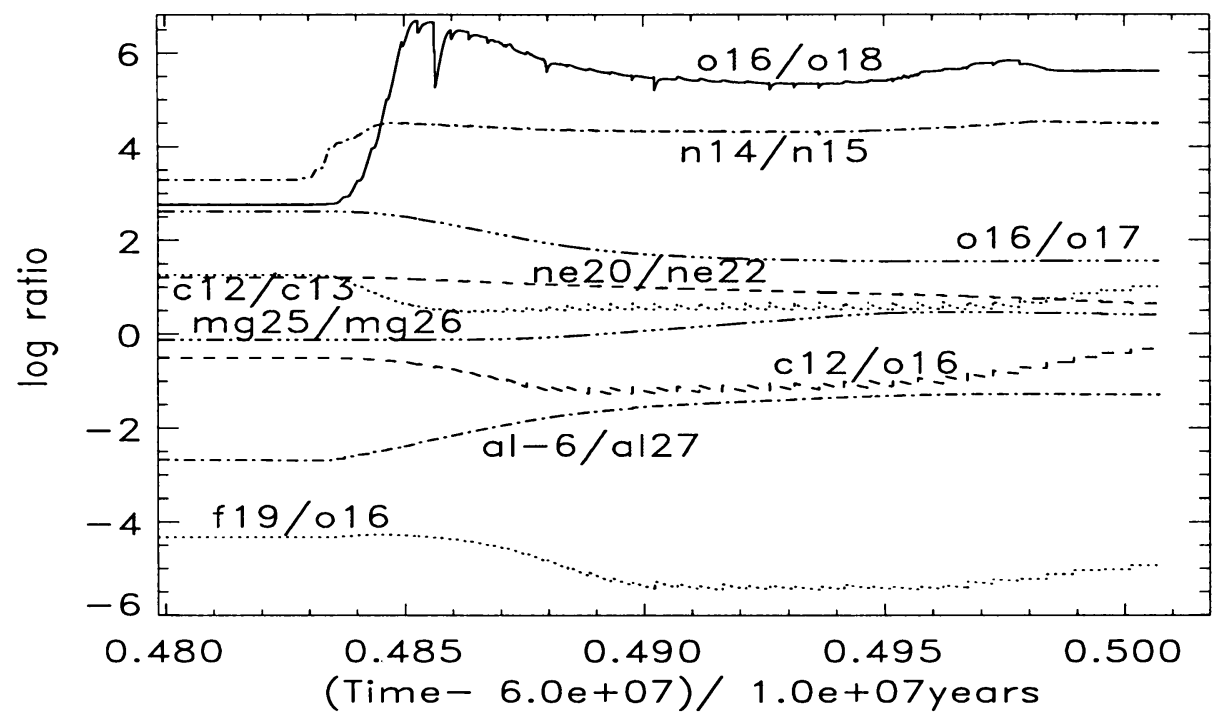

Figure 2. Selected isotopic ratios seen at the surface of the M6Z02 model during its thermally pulsing AGB evolution.

synthesis which occurs between the $30^{\text {th }}$ and $31^{\text {st }}$ pulses of M6Z02, being a typical interpulse phase showing HBB.

In the deepest layer of the convective envelope the temperatures are about 82 million $\mathrm{K}$, and there is hydrogen burning via the $\mathrm{CNO}$ cycle. Initially the ${ }^{12} \mathrm{C} /{ }^{13} \mathrm{C}$ ratio is out of equilibrium because of the ${ }^{12} \mathrm{C}$ added to the envelope by the previous dredge-up episode. Thus the ${ }^{13} \mathrm{C}$ rises and ${ }^{12} \mathrm{C}$ falls as these come into equilibrium. The convective turnover timescale is such that the entire envelope passes through the hot bottom many times during the interpulse phase, producing the equilibrium abundances of ${ }^{12} \mathrm{C}$ and ${ }^{13} \mathrm{C}$. Then both $\mathrm{C}$ isotopes fall as they are processed into ${ }^{14} \mathrm{~N}$. Further, at these high temperatures ${ }^{26} \mathrm{Mg}$ suffers substantial proton captures and produces ${ }^{27} \mathrm{Al}$. Also, the envelope ${ }^{19} \mathrm{~F}$ is destroyed by $(\mathrm{p}, \alpha)$ reactions. Thus it would appear that stars experiencing HBB should not show increases of both ${ }^{19} \mathrm{~F}$ and ${ }^{7} \mathrm{Li}$ simultaneously. This point has recently been made by Mowlavi et al. (1996), to whom we defer for details.

Figure 3 shows the region just below the convective envelope near the end of the interpulse phase - a region which has been processed by the hydrogen shell during the interpulse. First look at the ${ }^{13} \mathrm{C}$ profile. It leaves the hydrogen shell with $\log Y_{13} \simeq-4.9$, yet during the interpulse phase this burns totally via alpha captures, with a resulting increase in ${ }^{16} \mathrm{O}$. This was seen first in low-mass stars by Straniero et al. (1995) and later verified in more massive stars by Mowlavi et al. (1996). Some of the neutrons so 


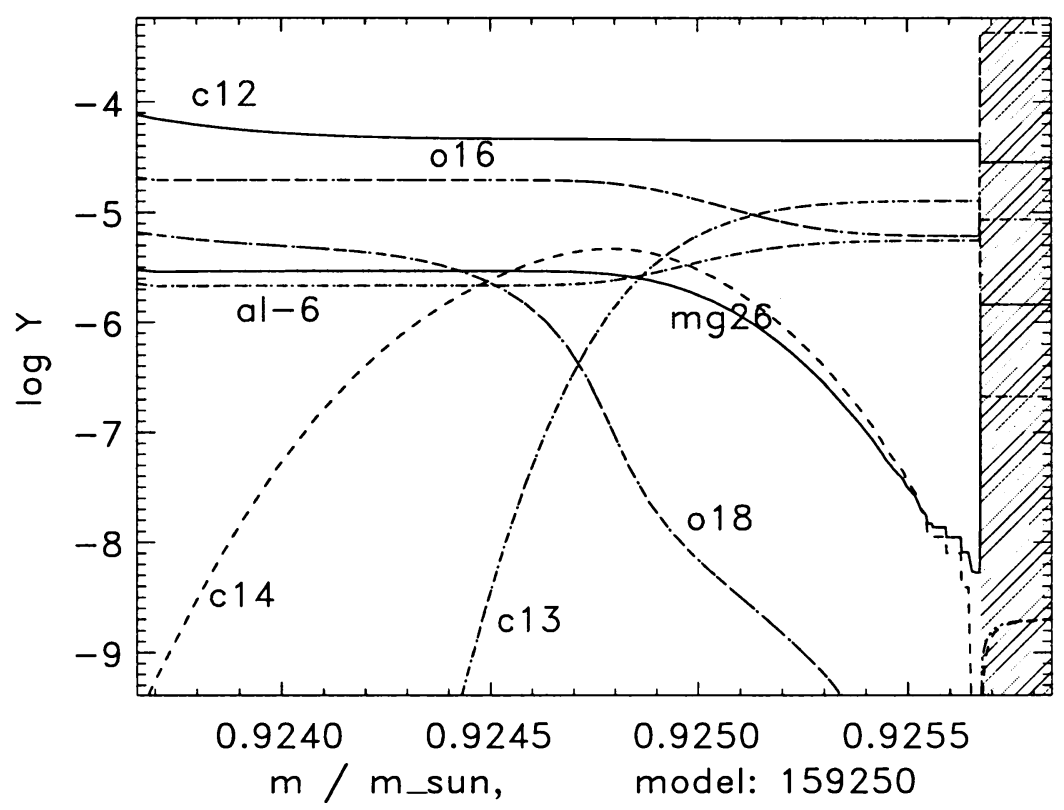

Figure 3. Compositions in a model near the end of the interpulse phase. The hatched region represents convection. "al-6" refers to the ground state of ${ }^{26} \mathrm{Al}$.

released are absorbed by the abundant ${ }^{14} \mathrm{~N}$ (not shown) to produce ${ }^{14} \mathrm{C}$ via $(\mathrm{n}, \mathrm{p})$ reactions, as discussed above. This ${ }^{14} \mathrm{C}$ also captures an alpha particle to produce the ${ }^{18} \mathrm{O}$ rise seen in Figure 3 . This will be absorbed by the next thermal pulse, to produce ${ }^{22} \mathrm{Ne}$ and hence neutrons (the subject of a subsequent paper). The hydrogen shell also produces ${ }^{27} \mathrm{Al}$ at the level $\log Y_{27} \simeq-5.3$ in the envelope, but it rises to -4.7 interior to the hydrogen shell, due to the proton captures on ${ }^{26} \mathrm{Mg}$ which occur there. When this material is added to the envelope after the next dredge-up phase it will enhance the surface composition of ${ }^{27} \mathrm{Al}$.

We have seen the surface composition changes for the first 43 pulses of the M6Z02 model. The same calculation is shown in Figures 4 and 5 for $Z=0.008$ and 0.004 , respectively. In each case the increase in ${ }^{14} \mathrm{~N}$ from $\mathrm{CN}$ cycling by $\mathrm{HBB}$ is quite apparent. As we have seen earlier, the ${ }^{18} \mathrm{O}$ is almost completely destroyed within the envelope as soon as HBB begins. Likewise, ${ }^{15} \mathrm{~N}$ is seen in equilibrium with ${ }^{14} \mathrm{~N}$ for most of the evolution. We also see in the figures the production of ${ }^{25} \mathrm{Mg},{ }^{26} \mathrm{Al}$ and ${ }^{27} \mathrm{Al}$, all due to the operation of the $\mathrm{Mg}$ - $\mathrm{Al}$ cycle both in the hot-bottomed envelope as well as in the hydrogen shell, with subsequent dredge-up.

Having discussed the M6Z02 case we can see the contrasts with the lower metallicity cases. Decreasing $Z$ causes higher temperatures for HBB. 


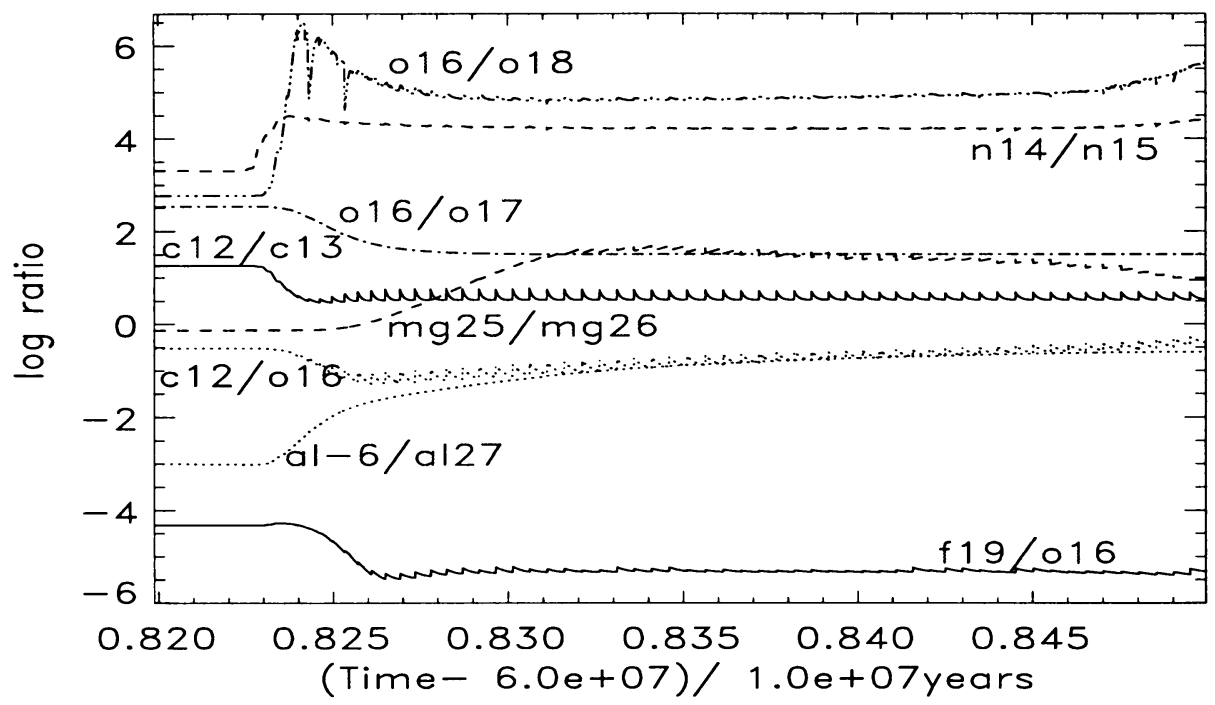

Figure 4. Surface composition for the M6Z008 model.

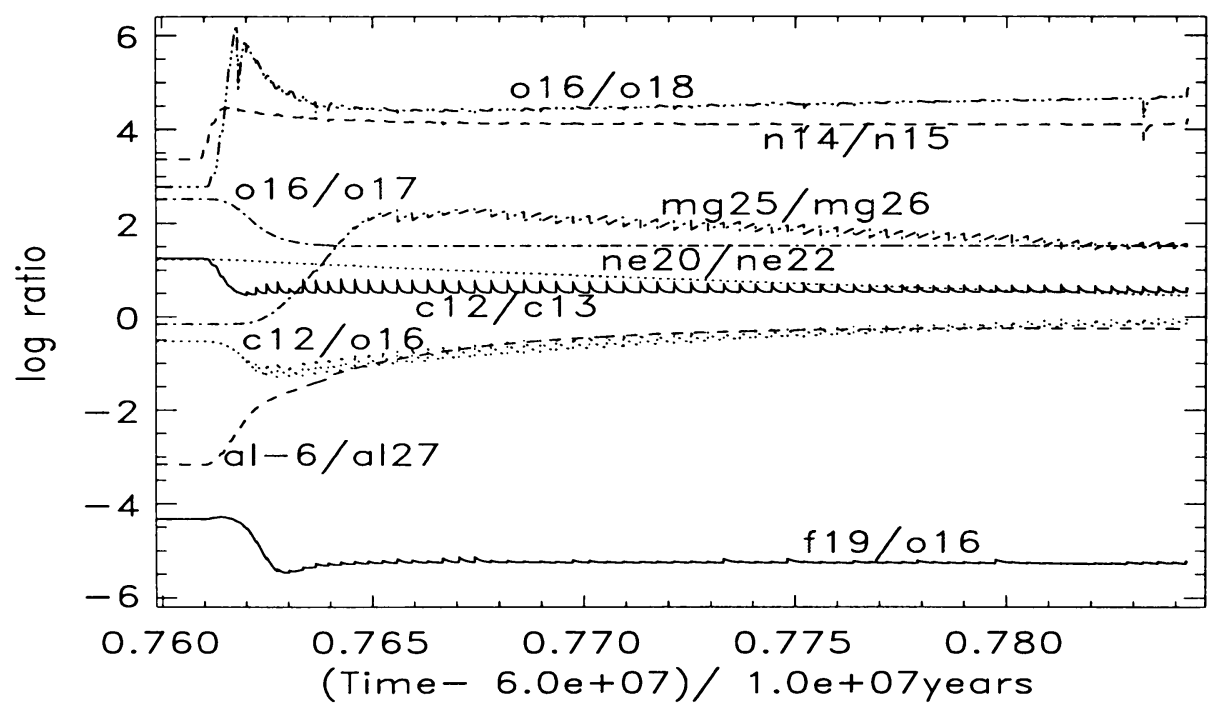

Figure 5. Surface composition for the M6Z004 model.

This is seen in the higher ratio of ${ }^{26} \mathrm{Al} /{ }^{27} \mathrm{Al}$ as $Z$ decreases. Note that with the most ${ }^{26} \mathrm{Al}$-rich meteorite grains (which reach values of ${ }^{26} \mathrm{Al} /{ }^{27} \mathrm{Al}$ up to 0.1 ; Zinner 1995), the current models are easily able to explain this measurement. A somewhat surprising result is the continued growth of ${ }^{12} \mathrm{C} /{ }^{16} \mathrm{O}$ during the HBB phase for the lower metallicity models. The dredge-up is 
so efficient at adding carbon to the envelope that despite the CN cycling, there is a continual increase in the ${ }^{12} \mathrm{C} /{ }^{16} \mathrm{O}$ ratio. The figures show that the ${ }^{12} \mathrm{C} /{ }^{16} \mathrm{O}$ ratios are close to unity (and the total $\mathrm{C} / \mathrm{O}$ will be higher because of the ${ }^{13} \mathrm{C}$ present). This raises the possibility of a population of very high luminosity carbon stars. At the end of the calculations shown, the M6Z008 case has $M_{\text {bol }}=-6.6$ and for M6Z004 we have $M_{\text {bol }}=-7.2$. These stars may not be visible, however, because of the high mass-loss rates seen as they near the end of their lifetimes. Nevertheless, we note the identification of high luminosity, high mass-losing carbon stars by van Loon et al. (2000) at this meeting.

\section{Conclusions}

We have presented a brief summary of the nucleosynthesis in three model stars of $6 M_{\odot}$ with $Z=0.02,0.008$ and 0.004 . We find very deep dredge-up occurs with the dredge-up parameter $\lambda$ approaching unity. We have calculated the detailed processing during $43(Z=0.02)$ to $66(Z=0.004)$ pulses, and found that the models match quite well many known constraints (e.g. first and second dredge-up isotopic ratios). Further, during the AGB evolution a combination of dredge-up and HBB leads to many surface composition changes. A full analysis of these models, as well as six others of differing mass, will form the subject of later papers.

We thank Arnold Boothroyd and Roberto Gallino for many useful discussions. This work was supported by the Australian Research Council, and the British Council.

\section{References}

Boothroyd, A. I. \& Sackmann, I.-J. 1992, ApJ, 393, L21

Cannon, R. C. 1993, MNRAS, 263, 817

Cannon, R. C., Frost, C. A., Lattanzio, J. C. \& Wood, P. R. 1998, in preparation

Frost, C. A. \& Lattanzio, J. C. 1996a, ApJ, 473, 383

Frost, C. A. \& Lattanzio, J. C. 1996b, in Stellar Evolution: What Should Be Done?, Proceedings of the 32nd Liège Colloquium, ed. A. Noels et al., p. 307

Groenewegen, M. A. T. \& de Jong, T. 1993, $A \mathcal{E} A, 267,410$

Käppeler, F., Wiescher, M., Giesen, U., Görres, J., Baraffe, I., El Eid, M., Raiteri, C. M., Busso, M., Gallino, R., Limongi, M. \& Chieffi, A. 1994, ApJ, 437, 396

Lattanzio, J. C., Frost, C. A., Cannon, R. C. \& Wood, P. R. 1996, Mem. Astron. Soc. Italia, 67, 729

Mowlavi, N., Jorissen, A. \& Arnould, M. 1996, $A \& A, 311,803$

Rogers, F. J. \& Iglesias, C. A. 1992, ApJ Supp., 79, 507

Straniero, O., Gallino, R., Busso, M., Chieffi, A., Raiteri, C. M., Limongi, M. \& Salaris, M. $1995, A p J, 440, \mathrm{~L} 85$

Thielemann, F.-K., Arnould, M. \& Truran, J. W. 1987, in Advances in Nuclear Astrophysics, ed. E. Vangioni-Flam et al. (Editions Frontiers: Gif-sur-Yvette), p. 525

van Loon, J. Th., Zijlstra, A. A., Whitelock, P. A., Loup, C. \& Waters, L. B. F. M. 2000, 
in IAU Symp. 177: The Carbon Star Phenomenon, ed. R.F. Wing (Kluwer), p. 145 Vassiliadis, E. \& Wood, P. R., 1993, ApJ, 413, 641

Zinner, E.K. 1995, in Nuclei in the Cosmos III, ed. M. Busso, R. Gallino and C. M. Raiteri, (AIP Press: New York), p. 567

\section{Discussion}

Frogel: In my talk the other day I ruled out HBB as being able to turn a C star back into an $\mathrm{M}$ star for 1-4 $M_{\odot}$. For the more massive stars that you are talking about, the problem in testing your results is that there are too few LMC clusters in the appropriate age range to give good enough statistics.

Lattanzio: So it's the perfect theory....

Frogel: Almost certainly you can rule out high-mass $\mathrm{C}$ stars contributing $50 \%$ of all AGB stars in the appropriate mass range, and maybe even at the $25 \%$ level. Beyond that there are not enough real stars of known physical properties in the LMC.

Lattanzio: You are right, of course. There cannot be many of these stars, even if the models are correct. Also, as these "late conversion" C stars are experiencing rapid mass loss, they may not be visible at all!

Ake: Can you tell us what happens with $\mathrm{Li}$ ?

Lattanzio: It should be very efficiently produced by stars in the mass range of about 4-6 $M_{\odot}$, as shown by Boothroyd \& Sackmann (1992, ApJ, $393, \mathrm{~L} 21)$. Once all the ${ }^{3} \mathrm{He}$ has been used in producing this $\mathrm{Li}$, then the HBB succeeds in destroying the $\mathrm{Li}$, so that the Li-rich period is a temporary (but not particularly short) phase of evolution. Boothroyd has also been looking at the possibility that deep mixing on the AGB can produce $\mathrm{Li}$ in low-mass stars. Although this relies on parametrized calculations, the required values of the parameters are quite reasonable. Of course, we really need to understand properly what drives this proposed extra-mixing. But that is a very complicated problem.

Gustafsson: You did not mention sodium. There is some emprical evidence for an intermediate-mass sodium source. Do your models produce any?

Lattanzio: There is no significant change in the ${ }^{23} \mathrm{Na}$ abundance on the AGB: a small increase is seen at second dredge-up and then a small decrease during HBB (at least, with the Caughlan \& Fowler 1988 rates). There is significant production of ${ }^{21} \mathrm{Na}$, but it is always at a very low level, about $\log \mathrm{Y} \approx-10$, which is unobservable, I believe! 
Jorissen: Could ${ }^{14} \mathrm{~N}$ be used as a diagnostic of HBB?

Lattanzio: I think that is a question an observer could better answer. ${ }^{14} \mathrm{~N}$ is quite abundant, so you would need good quantitative analysis, I think. Maybe ${ }^{14} \mathrm{~N} /{ }^{15} \mathrm{~N}$ is more useful. We know from measurements of this ratio in meteorite grains that there is a wide spread in the value found - wider than the models seem to give.

Little-Marenin: What is the minimum mass for HBB? And does this mean that this method only produces high-luminosity J stars?

Lattanzio: $\mathrm{HBB}$ seems to occur in stars of about $4-6 M_{\odot}$, so the ${ }^{13} \mathrm{C}$ production by this mechanism would only occur for relatively high luminosities, as you say. But if there is some deep mixing, and I think that the evidence now is that there probably is, then maybe this can produce enrichments of ${ }^{13} \mathrm{C}$ just as found on the first giant branch in globular cluster stars. 